Abellán, J.; Sáez-Gallego, N.M.; Savelsbergh, G.J.P. y Contreras, O.R. (2019) La interceptación de un lanzamiento de córner desde la teoría de los limitadores / The Interception of a Corner Kick from the Contraints-Led Perspective. Revista Internacional de Medicina y Ciencias de la Actividad

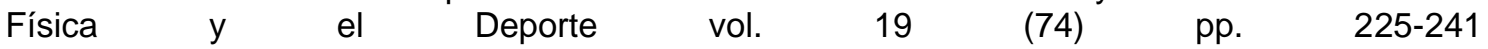
Http://cdeporte.rediris.es/revista/revista74/artintercepcion1016.htm DOI: http://doi.org/10.15366/rimcafd2019.74.004

\title{
ORIGINAL
}

\section{LA INTERCEPCIÓN DE UN LANZAMIENTO DE CÓRNER DESDE LA TEORÍA DE LOS LIMITADORES}

\section{INTERCEPTION OF A CORNER KICK FROM THE CONTRAINTS-LED APROACH}

\author{
Abellán, J.. ; Sáez-Gallego, N.M.'; Savelsbergh, G.J.P. ${ }^{2}$ y Contreras, O.R. ${ }^{1}$ \\ ${ }^{1}$ Facultad de Educación, Universidad de Castilla-La Mancha (España) jorge.abellan@uclm.es, \\ nieves.saez@uclm.es, Onofre.cjordan@uclm.es \\ ${ }^{2}$ Research Institute MOVE, Faculty of Human Movement Sciences, VU University Amsterdam, \\ (The Netherlands) g.j.p.savelsbergh@vu.nl
}

\section{AGRADECIMIENTOS}

La presente investigación se llevó a cabo mientras el primer autor y la segunda autora disfrutaban de una beca FPU (Formación del Profesorado Universitario) otorgada por el Ministerio de Educación, Cultura y Deporte del Gobierno de España. Los autores quieren agradecer la participación del Dr. Rouwen Cañal-Bruland en la versión inicial de este trabajo.

Código UNESCO / UNESCO code: 6199 Otras especiales psicológicas: Psicología del deporte/ Others: Sport Psychology

Clasificación Consejo de Europa / Council of Europe classification: 15 Psicología del deporte/ Sport Psychology

Recibido 23 de mayo de 2015 Received May 23, 2015

Aceptado 18 de noviembre 2017 Accepted November 2017

\section{RESUMEN}

El comportamiento motor surge de la continua interacción entre tres limitadores (organismo, entorno y tarea), que nunca actúan de forma aislada. Este artículo estudia su efecto en el rendimiento, comportamiento motor y comportamiento visual de porteros de fútbol durante el lanzamiento de córner. 31 participantes, divididos en tres grupos en función del nivel de juego, intentaron atrapar el balón procedente del lanzamiento de córner en dos situaciones (estática y dinámica), mientras que se registraron sus movimientos oculares. Entre los resultados se observa que los expertos tienen un rendimiento más estable, mientras que los otros grupos rinden peor en la situación más difícil; que los expertos realizan un inicio más tardío de la carrera hacia el balón y un patrón 
motor más rápido para atraparlo; y que la información contenida en los jugadores implicados no es relevante, ya que los porteros dedican valores cercanos al $0 \%$ del tiempo total a su fijación.

PALABRAS CLAVE: comportamiento motor, comportamiento visual, porteros de fútbol.

\begin{abstract}
Motor behavior arises from the continuous interaction between three constraints (organism, environment and task), which never act in isolation. This paper studies the effect of the constraints on the performance, motor behavior and visual search behavior of soccer goalkeepers during the corner kick. 31 participants, divided into three groups depending on the level of play, tried to catch the ball out of a corner kick in two situations (static and dynamic), while their eye movements were recorded. Among the results it is observed that the experts have a more stable performance, while the other groups perform worse in the most difficult situation; that the experts make a later start of their run up towards the ball and a faster motor pattern to catch it; and that the that the information of the players involved is not relevant, goalkeepers dedicate values close to $0 \%$ of their visual total time to them.
\end{abstract}

KEY WORDS: movement behaviour, visual search, soccer goalkeepers.

\title{
INTRODUCCIÓN
}

La Teoría de los Sistemas Dinámicos (Berstein, 1967) ha sido un marco de trabajo funcionalista para entender la coordinación neurobiológica del movimiento. Desde esta perspectiva, el individuo está compuesto por muchos subsistemas que interactúan formando patrones de comportamiento eficaces en una determinada situación (Handford, Davids, Bennett y Button, 1997). Las diferentes configuraciones que los elementos independientes de estos subsistemas pueden tomar se denominan grados de libertad (Berstein, 1967).

Con el objetivo de entender como el rendimiento difiere en función de la habilidad seleccionada de una determinada modalidad deportiva, y de acuerdo con Newell (1986), es importante identificar los limitadores que constriñen la emergencia de patrones motores orientados a la consecución de un objetivo. Newell (1986) propuso tres categorías de limitadores: los limitadores del organismo, limitadores relacionados con el propio individuo; los limitadores del entorno, externos al individuo; y los limitadores de la tarea, centrados en el objetivo de la acción (para ampliar la información consultar Newell \& Ranganathan, 2009). De acuerdo con este enfoque, los tres tipos de limitadores interactúan juntos, generando acoples de percepción-acción. Por ello, en un contexto deportivo, los limitadores de la tarea, del entorno o del organismo nunca actúan en solitario. 
Tradicionalmente, el efecto de los limitadores en el rendimiento y el comportamiento motor de los atletas se ha estudiado creando diferentes situaciones de juego (Araújo, Davids \& Travassos, 2012; Correia et al., 2012; Vilar, Correia, Araújo, Vilar \& Davids, 2013). Uno de los ejemplos es la investigación centrada en el efecto de los limitadores de la tarea en el comportamiento visual y la toma de decisiones en jóvenes jugadores de fútbol realizada por Vaeyens, Lenoir, Williams, Mazyn y Philippaerts (2007). Utilizaron videos simulados en cinco situaciones diferentes (2 vs. 1, 3 vs. 1, 3 vs. 2, 4 vs. 3 y 5 vs. 3) y como resultado obtuvieron que los grupos de jugadores de fútbol (de categorías regional, sub-élite y élite) eran más rápidos y precisos en el test de toma de decisiones que el grupo de participantes que no eran jugadores de fútbol. En un estudio posterior (Vaeyens, Lenoir, Williams \& Philippaerts, 2007), los participantes se ordenaron en función de su rendimiento en el test de toma de decisiones. Los jugadores exitosos presentaron un comportamiento visual considerado como más pertinente. En las situaciones de análisis más complejas, con más jugadores involucrados ( 3 vs. 2 y 4 vs. 3), las diferencias entre participantes exitosos y no exitosos se incrementaron. Posteriormente, Correia et al. (2012) estudiaron la toma de decisiones de jugadores de rugby manipulando la posición de partida de los defensores en una situación de 1 vs. 2. Una distancia más corta entre los defensores fue identificada como el comportamiento defensivo más efectivo, lo que significa que la situación de los defensas limita el comportamiento de los atacantes.

Vilar, Araújo, Davids, Correia y Esteves (2012) investigaron la influencia de los oponentes en la toma de decisiones de los jugadores atacantes durante acciones de lanzamiento a puerta en fútbol sala. La velocidad de movimiento de los oponentes limita la toma de decisiones durante la acción de tiro. Los resultados muestran que el defensor más cercano y el portero son significativamente más lentos cuando son capaces de interceptar el balón. Headrick et al. (2012) investigaron los limitadores espacio-temporales en una situación 1 vs. 1 en fútbol. Los resultados muestran que la proximidad a la portería influye en el rendimiento de los jugadores de fútbol.

Por otra parte, y centrándonos en la acción estudiada en el presente artículo, se ha venido investigando el efecto de los limitadores en el comportamiento motor, visual y en el rendimiento de los porteros cuando deben atrapar balones procedentes de lanzamientos de córner. En los estudios previos, los limitadores de esta acción se han manipulado uno a uno (Abellán, Savelsbergh, Contreras y Vila-Maldonado, 2016) o dos a dos (Abellán, SáezGallego y Contreras, 2015; Abellán, Sáez-Gallego, Vila-Maldonado y Contreras, 2017). En Abellán et al. (2016) se realizó una definición de los limitadores de la tarea. Los fallos en la intercepción de un lanzamiento de córner (que fue considerado como el objetivo de la acción) se atribuyeron a un comienzo demasiado temprano de la carrera previa y a un patrón coordinativo pobre e inadecuado de las acciones del salto y del blocaje por parte del portero. De manera que los aciertos en el blocaje se producían cuando los porteros comenzaban a moverse, saltar y mover sus manos más cerca del final de la acción. Adicionalmente, en este estudio se obtuvieron datos del comportamiento visual, que mostraron que la zona informativa más relevante fue el balón. 
En Abellán et al. (2015) se analizó el efecto de los limitadores del entorno (que se estudiaron junto a los de la tarea), creando dos situaciones diferentes en las que los porteros debían tratar de blocar balones que procedían de lanzamientos de córner: una de ellas con adversarios en la zona de acción del portero y la otra sin ellos. Los resultados en rendimiento mostraron que el porcentaje de acierto no difería entre las dos situaciones. Sin embargo, si se encontraron diferencias en el comportamiento motor, específicamente en el momento de inicio de la carrera, ya que cuando los porteros debían atrapar un lanzamiento de córner con jugadores dentro de su zona de acción comenzaban a moverse significativamente antes. No obstante, la ausencia de diferencias en el grupo de porteros que realiza ambas situaciones de análisis hace que los resultados deban ser tratados con cautela.

Finalmente, Abellán et al. (2017) estudiaron el efecto de los limitadores del organismo (junto con los de la tarea) agrupando a los porteros en función de su éxito en el blocaje de lanzamientos de córner. En este estudio, los porteros más exitosos conseguían su rendimiento superior mediante un inicio más tardío del movimiento de manos y del atrape del balón que los porteros menos exitosos.

En el presente trabajo, igual que durante un lanzamiento de córner en un partido real, porteros con diferente nivel de juego (limitador del organismo) tienen que atrapar balones procedentes de lanzamientos de córner (limitador de la tarea) en dos situaciones diferentes de análisis creadas (limitador del entorno). En este contexto, los porteros deberán seleccionar la solución óptima de la tarea de la variedad de opciones posibles. El comportamiento de los porteros durante la acción no va a depender sólo de los limitadores del entorno, por ejemplo, adversarios moviéndose en su zona de acción, sino también de sus experiencias pasadas y del éxito que tuvieron en ellas (Davids, Williams, Button \& Court, 2001). La principal idea es conseguir identificar la influencia de estos limitadores para la consecución del éxito en esta acción determinada, con el objetivo de que los entrenadores o maestros puedan guiar el proceso en aprendizaje mediante la creación de tareas de entrenamiento adecuadas (Savelsbergh, Verheul, Van der Kamp \& Marple-Horvat, 2007).

Con todo ello, el presente trabajo tiene como objetivo analizar las diferencias en el rendimiento, en el comportamiento motor y en el comportamiento visual de tres grupos de jóvenes porteros con diferente nivel de juego (expertos, intermedios-altos e intermedios) durante la intercepción de balones procedentes de lanzamientos de córner en dos situaciones: la situación estática, en la que existen jugadores inmóviles en la zona de acción del portero, y la situación dinámica, en la que existen jugadores en movimiento en dicha zona. Se parte de la hipótesis de que los porteros expertos tendrán un rendimiento superior, con un mayor porcentaje de blocajes, y utilizarán un patrón coordinativo diferente al de sus compañeros de menor nivel. En cuanto al análisis del comportamiento visual, y teniendo en cuenta los resultados de estudios previos (Abellán et al., 2016; Sáez-Gallego, Vila-Maldonado, Abellán y Contreras, 2013; Vila-Maldonado, Sáez-Gallego, Abellán y Contreras, 2012), se prevé que el balón sea de nuevo la zona informativa más relevante. 


\section{MÉTODO}

\section{Participantes}

Se utilizó una muestra intencional compuesta por treinta y un porteros de categoría juvenil (sub-19). Los porteros que jugaban en la categoría más alta del fútbol juvenil español (División de Honor) fueron considerados el grupo experto $(N=10$, edad media $=18,2 \pm 0,63$ años, experiencia $=10,5$ años $)$. Los porteros que jugaban en la segunda categoría del fútbol juvenil español (Juvenil Nacional) fueron considerados el grupo intermedio-alto $(N=11$, edad media $=16,45 \pm 0,69$ años, experiencia $=7,27$ años). Los porteros que jugaban en la tercera categoría del fútbol juvenil español (Juvenil Provincial) fueron considerados el grupo intermedio ( $N=10$, edad media $=16,6 \pm 0,84$ años, experiencia $=7$ años). Cada participante recibió una invitación a participar en el estudio, explicando los objetivos de este y obteniendo el consentimiento informado. En el caso de los participantes menores de edad se obtuvo el consentimiento de los padres 0 tutores. Todos los participantes, así como sus entrenadores, tomaron parte en el estudio voluntariamente.

\section{Material}

El comportamiento motor de los porteros fue registrado mediante el uso de dos videocámaras (Sony Handycam DCR-HC42E PAL), una de ellas situada en la esquina del área opuesta al lugar de lanzamiento del córner y la otra situada en línea con el punto de penalti y la otra cámara (Figura 1).

El comportamiento visual fue grabado con un sistema de registro de la mirada (ASL Mobile Eye). Este sistema está compuesto por dos cámaras montadas en un soporte en forma de gafas. Una de las cámaras graba el espejo en el que se refleja la pupila, con el objetivo de registrar los movimientos oculares. La segunda cámara es la encargada de grabar la escena. El sistema informático combina las dos señales en un solo video que muestra la escena con un cursor que indica el punto en el que el participante detiene la mirada. Antes del comienzo de la prueba, se realizó la calibración del instrumento para cada participante. Además, se comprobó el estado de la calibración cada cinco lanzamientos, para ello se pidió a los participantes que miraran a cinco puntos situados en un panel detrás de la portería.

Los materiales utilizados habían sido empleados previamente en investigaciones anteriores con similares objetivos (Abellán, et al., 2015; 2016; 2017).

\section{Procedimiento}

El protocolo utilizado en este estudio está de acuerdo con las exigencias de la Declaración de Helsinki. Además, se siguieron los procedimientos utilizados previamente por Abellán et al. (2015; 2016; 2017). 
De un total de 620 lanzamientos de córner, finalmente 602 fueron analizados. Se indicó a los porteros que debían atrapar los balones procedentes de lanzamientos de córner del lado izquierdo, debido a los resultados que no encontraron diferencias en función del lado de lanzamiento en el trabajo previo de Abellán et al. (2016). Cada uno de los intentos comenzaba con el portero situado en la línea de gol (Figura 1). Dos jugadores diestros realizaban los lanzamientos, rotando cada dos golpeos. Los lanzadores tenían el mismo nivel competitivo que los porteros participantes. Tanto a los lanzadores como a los porteros se les indicó que debían actuar como lo harían en un partido real, teniendo en cuenta que su comportamiento no pusiera en peligro su propia integridad ni la del sistema de registro de la mirada. El destino de los lanzamientos de córner fue manipulado de acuerdo con los resultados obtenidos por Abellán et al. (2016). Todos los lanzamientos se dirigieron a un área específica formada por un rectángulo de $9,32 \mathrm{~m}$. de alto y $11 \mathrm{~m}$. de ancho (ver Figura 1). Las situaciones de análisis fueron balanceadas para cada participante. Dicha zona de lanzamiento ya había sido previamente utilizada en Abellán et al. $(2015 ; 2017)$ Se realizaron un total de 20 lanzamientos en la situación de análisis estática y la dinámica (10 en cada una):

\section{Situación estática}

Los porteros debían tratar de atrapar un lanzamiento de córner desde el lado izquierdo mientras dos jugadores se encontraban dentro de su zona de acción. Estos jugadores estaban situados enfrente de cada uno de los postes de la portería y encima de la línea del área pequeña. Los jugadores no podían tocar el balón o moverse, tan sólo actuaban como referencia (Figura 1 sección A).

\section{Situación dinámica}

Los porteros debían tratar de atrapar un lanzamiento de córner desde el lado izquierdo mientras dos jugadores se encontraban dentro de su zona de acción. Estos jugadores estaban situados enfrente de cada uno de los postes de la portería y a la altura del punto de penalti. En el momento del golpeo los jugadores comenzaban a correr en línea recta hasta llegar a la línea del área pequeña (Figura 1 sección B). Los jugadores no podían tocar el balón, tan sólo actuaban como referencia.

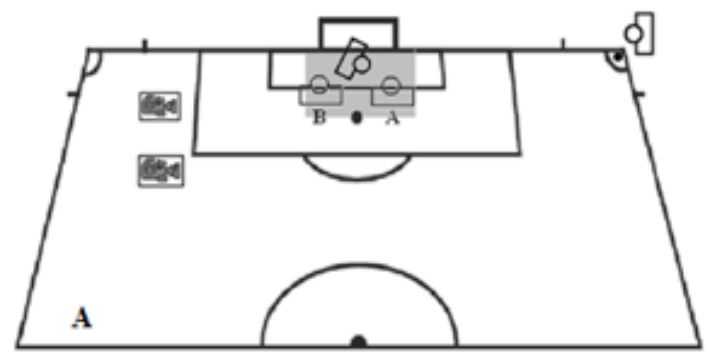

Jugador/portero

$$
\text { Balón }
$$

Posición de la cámara

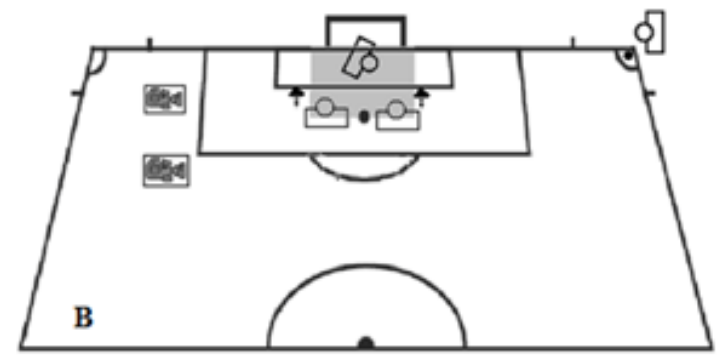

Destino del balón

Figura 1. Vista del diseño experimental con las dos situaciones de análisis creadas, estática (A) y dinámica (B). 
Variables y análisis estadístico

Los videos resultantes (Figura 1) fueron analizados fotograma a fotograma por el mismo investigador. El investigador anotaba en una hoja de registro el comienzo y el final de las variables analizadas de acuerdo con el programa de análisis de video VirtualDub. Del análisis se obtuvieron tres tipos distintos de datos: datos del rendimiento, datos del comportamiento motor y datos del comportamiento visual. Los datos se analizaron realizando una ANOVA de medidas repetidas, tomando el Grupo (expertos, intermedios-altos e intermedios) x Situación (Estática vs. Dinámica) con un nivel de significación de $p \leq 0,05$. Se realizaron pruebas $T$ pareadas como análisis post-hoc con el objetivo de comparar las diferencias significativas.

Variable independiente

Como variable independiente se consideró el nivel competitivo (dentro de la misma categoría sub-19), comparando los resultados de las variables dependientes en base a la pertenencia a uno de estos tres grupos de análisis:

Grupo expertos: porteros que competían en la liga División de Honor Juvenil, máxima categoría española juvenil.

Grupo intermedios-altos: porteros que competían en la liga Juvenil Nacional, una categoría por debajo de la máxima categoría juvenil española.

Grupo intermedios: porteros que competían en la liga Juvenil Provincial, dos categorías por debajo de la máxima categoría juvenil española.

Variables dependientes

Datos del rendimiento

El rendimiento de los porteros se analizó a partir de la siguiente variable:

Porcentaje de fallos: es el porcentaje de lanzamientos en los que el portero no consiguió atrapar el balón.

\section{Comportamiento motor}

El comportamiento motor de los porteros se analizó por medio de los aspectos temporales del movimiento desde dos puntos de referencia: desde el momento del golpeo del balón por parte del lanzador y desde el momento del blocaje por parte del portero. Todas las variables fueron calculadas en segundos.

Las variables analizadas desde el momento del golpeo son: 
El tiempo de la carrera: es el intervalo de tiempo entre el golpeo del balón por parte del lanzador y el momento en el que el portero comienza a moverse.

El tiempo del salto: es el intervalo de tiempo entre el golpeo del balón por parte del lanzador y el momento en el que el portero comienza a saltar.

El tiempo del movimiento de las manos: es el intervalo de tiempo entre el golpeo del balón por parte del lanzador y el momento en el que el portero comienza a mover las manos en dirección al balón.

El tiempo del blocaje: es el intervalo de tiempo entre el golpeo del balón por parte del lanzador y el blocaje del balón por parte del portero. Cuando los porteros no consiguen atrapar el balón, el tiempo del blocaje se obtiene del momento en el que los porteros tocan el balón o el momento en el que el balón pasa a la altura de sus manos.

Las variables analizadas desde el momento del blocaje son:

El tiempo del movimiento de las manos: es el intervalo de tiempo entre el comienzo del movimiento de las manos por parte del portero y el blocaje del balón.

El tiempo del salto: es el intervalo tiempo entre el comienzo del salto por parte del portero y el blocaje del balón.

El tiempo de la carrera: es el intervalo de tiempo entre el comienzo de la carrera por parte del portero y el blocaje del balón.

El tiempo del vuelo del balón: es el intervalo de tiempo entre el golpeo del balón por parte del lanzador y el blocaje del balón por parte del portero.

\section{Comportamiento visual}

Debido a problemas con la calibración, se obtuvieron datos del comportamiento visual de 15 porteros ( 5 expertos, 5 intermedios-altos y 5 intermedios). El sistema de registro de la mirada se utilizó para analizar el comportamiento visual de los porteros mientras trataban de atrapar balones procedentes de lanzamientos de córner. Se seleccionaron dos periodos temporales para analizar. Estos periodos fueron:

Periodo de pre-contacto: es el intervalo de tiempo de un segundo entre la carrera del lanzador y el golpeo del balón (la localización carrera del lanzador fue registrada en este periodo).

Periodo del vuelo del balón: es el intervalo de tiempo entre el golpeo del balón por parte del lanzador y el movimiento en el que el portero inicia la carrera para atrapar el balón (la localización balón fue registrada en este periodo). 
Para ambas variables se calculó el porcentaje de tiempo mirando a las localizaciones con respecto al porcentaje de tiempo total del periodo.

\section{RESULTADOS}

\section{Datos del rendimiento}

En la Tabla 1 se presenta el porcentaje de fallos en el blocaje, separado para cada grupo y situación de análisis (estática y dinámica). El total de fallos se incluye con el objetivo de completar la información sobre el rendimiento de los porteros.

Tabla 1. Porcentaje de fallos en el blocaje de los porteros, divididos en grupos, en cada una de las situaciones de análisis creadas. El asterisco $\left({ }^{*}\right)$ indica diferencias significativas para $p \leq 0,05$.

$$
\text { Expertos Intermedios-altos Intermedios }
$$

\begin{tabular}{lccc}
\hline Estática $^{*}$ & 6 & 7,76 & 18,33 \\
Dinámica* $^{*}$ & 2 & 12,83 & 21,29 \\
Total $^{*}$ & 4,03 & 10,31 & 19,96 \\
\hline
\end{tabular}

Tal y como se puede observar en la Tabla 1, el grupo de porteros expertos tiene un menor porcentaje de fallos en las dos situaciones de análisis. Además, el grupo intermedios-altos tiene menos porcentaje de fallos que el grupo intermedios en cada situación de análisis.

El ANOVA de medidas repetidas, tomando el grupo (expertos, intermedios-altos e intermedios) x la situación (estática vs. dinámica) revela diferencias significativas en el porcentaje de fallos $[F(1,28)=11,238, p=0,000]$.

La prueba T, utilizada como post-hoc, revela diferencias significativas al comparar a los porteros Expertos vs. Intermedios-altos. El grupo de Expertos cometió significativamente menos fallos en la condición dinámica $[t(19)=3,46, p$ $=0,003]$ y en el total $[t(19)=2,78, p=0,012]$.

Asimismo, la prueba $T$ reveló diferencias significativas al comparar los grupos Expertos vs. Intermedios, El grupo expertos fue más exitoso que el grupo intermedios en las dos situaciones y en el total. Los resultados para cada una de las situaciones fueron los siguientes: $t(18)=2,76, p=0,013$ para la situación estática; $t(18)=3,85, p=0,001$ para la situación dinámica; y $t(18)=4,36, p=$ 0,000 para el total (situaciones dinámica y estática juntas).

Por último, la prueba T también reveló diferencias significativas entre los grupos intermedios-altos e intermedios. El grupo intermedios-altos fue más exitoso que el intermedios y obtuvo diferencias significativas en la situación estática $[t(19)=2,47, p=0,023]$ y en el análisis total $[t(19)=2,46, p=0,024]$. 


\section{Aspectos temporales del movimiento}

Los valores medios (en segundos) del comportamiento motor, analizados desde el momento del golpeo, se presentan en la Tabla 2.

Tabla 2. Medias y desviación típica del comportamiento motor (en segundos) analizados desde el momento del golpeo. El asterisco $\left(^{*}\right)$ indica diferencias significativas para $p \leq 0,05$.

\begin{tabular}{|c|c|c|c|c|c|c|}
\hline & \multicolumn{2}{|c|}{ Expertos } & \multicolumn{2}{|c|}{ Intermedios-altos } & \multicolumn{2}{|c|}{ Intermedios } \\
\hline & Estática & Dinámica & Estática & Dinámica & Estática & Dinámica \\
\hline Carrera & $0,34 \pm 0,08$ & $0,33 \pm 0,07$ & $0,31 \pm 0,12$ & $0,31 \pm 0,10$ & $0,31 \pm 0,06$ & $0,29 \pm 0,14$ \\
\hline Salto* & $1,50 \pm 0,22$ & $1,51 \pm 0,23$ & $1,60 \pm 0,14$ & $1,56 \pm 0,09$ & $1,70 \pm 0,19$ & $1,73 \pm 0,08$ \\
\hline Movimiento de las manos ${ }^{*}$ & $1,55 \pm 0,20$ & $1,55 \pm 0,21$ & $1,63 \pm 0,14$ & $1,60 \pm 0,11$ & $1,75 \pm 0,13$ & $1,71 \pm 0,10$ \\
\hline Blocaje* & $1,82 \pm 0,19$ & $1,82 \pm 0,20$ & $1,87 \pm 0,14$ & $1,85 \pm 0,11$ & $2,04 \pm 0,13$ & $1,96 \pm 0,15$ \\
\hline
\end{tabular}

Los porteros expertos comenzaron su carrera previa más tarde que sus compañeros intermedios-altos e intermedios, sin embargo, realizaron antes que ellos el resto de las variables analizadas: tiempo del salto, comienzo del movimiento de las manos y el blocaje.

Se realizó un ANOVA de medidas repetidas para cada variable por separado, teniendo en cuenta el Grupo (expertos, intermedios-altos e intermedios) x la Situación (estática y dinámica). La prueba reveló diferencias significativas para el tiempo del salto $[F(1,28)=4,94, p=0,015]$, el comienzo del movimiento de las manos $[F(1,28)=3,78, p=0,035]$ y el momento del blocaje $[F(1,28)=4,65, p=0,018]$.

La prueba $T$ como análisis post-hoc para el salto reveló diferencias significativas entre el grupo expertos y el grupo intermedios en las situaciones de análisis estática [t(18) $=2,22, p=0,039]$ y dinámica $[t(18)=2,81, p=0,012]$, y entre los grupos intermedios-altos e intermedios en la situación dinámica [t(19) $=4,65, p=0,000]$. El grupo de expertos iniciaba su salto significativamente antes que los otros dos grupos, mientras que el grupo intermedios-altos lo hacía antes que el grupo de porteros intermedios.

La prueba T como análisis post-hoc para el tiempo de movimiento de las manos reveló diferencias significativas entre los grupos expertos e intermedios en las dos situaciones analizadas: estática $[t(18)=2,69, p=0,012]$ y dinámica $[t(18)=2,15, p=0,045]$. También se encontraron diferencias significativas entre los grupos intermedios-altos e intermedios en la situación dinámica $[t(19)=2,23$, $p=0,038]$. Los porteros expertos e intermedios-altos comenzaron a mover sus manos para tratar de atrapar el balón significativamente antes que los porteros intermedios.

Respecto al blocaje, el análisis post-hoc realizado con la prueba $T$ ha mostrado diferencias significativas entre los porteros expertos y los porteros intermedios en la situación de análisis estática $[t(18)=3,1, p=0,006]$. Además, 
se encontraron diferencias entre los grupos intermedios-altos e intermedios en las situaciones estática $[t(19)=3,02, p=0,007]$ y dinámica $[t(19)=2,46, p=$ $0,024]$. Los porteros intermedios intentaban atrapar el balón significativamente más tarde que los porteros expertos e intermedios-altos.

Los valores medios (en segundos) del comportamiento motor, analizados desde el momento del blocaje, se presentan en la Tabla 3.

Tabla 3. Medias y desviación típica del comportamiento motor (en segundos) analizados desde el momento del blocaje. El asterisco $\left(^{*}\right)$ indica diferencias significativas para $p \leq 0,05$.

\begin{tabular}{|c|c|c|c|c|c|c|}
\hline & \multicolumn{2}{|c|}{ Expertos } & \multicolumn{2}{|c|}{ Intermedios-altos } & \multicolumn{2}{|c|}{ Intermedios } \\
\hline & Estática & Dinámica & Estática & Dinámica & Estática & Dinámica \\
\hline Movimiento de las manos & $0,26 \pm 0,03$ & $0,27 \pm 0,04$ & $0,24 \pm 0,06$ & $0,25 \pm 0,05$ & $0,29 \pm 0,06$ & $0,26 \pm 0,04$ \\
\hline Salto* & $0,33 \pm 0,04$ & $0,33 \pm 0,04$ & $0,29 \pm 0,04$ & $0,30 \pm 0,04$ & $0,30 \pm 0,08$ & $0,25 \pm 0,06$ \\
\hline Carrera* & $1,45 \pm 0,24$ & $1,49 \pm 0,22$ & $1,56 \pm 0,23$ & $1,54 \pm 0,16$ & $1,74 \pm 0,14$ & $1,67 \pm 0,13$ \\
\hline Vuelo del balón* & $1,81 \pm 0,19$ & $1,82 \pm 0,20$ & $1,87 \pm 0,14$ & $1,85 \pm 0,11$ & $2,04 \pm 0,13$ & $1,96 \pm 0,09$ \\
\hline
\end{tabular}

Se realizó un ANOVA de medidas repetidas para cada variable por separado, teniendo en cuenta el Grupo (expertos, intermedios-altos e intermedios) x la Situación (estática y dinámica). Los resultados muestran un efecto significativo del grupo para el tiempo de movimiento de las manos $[F(1,28)=3,85, p=0,035]$, el momento de la carrera $[F(1,28)=4,33, p=0,023]$ y el tiempo del vuelo del balón $[F(1,28)=4,7, p=0,017]$.

La prueba T como análisis post-hoc muestra diferencias significativas para el momento del salto entre los grupos expertos e intermedios $[t(18)=3,66, p=$ $0,002]$ y entre los grupos intermedios-altos e intermedios $[t(19)=2,23, p=0,038]$ en la situación dinámica. Los porteros intermedios realizaron el salto más cerca del blocaje del balón en comparación con los porteros expertos e intermediosaltos en la situación dinámica.

Respecto a la variable carrera, el análisis post-hoc realizado con la prueba $\mathrm{T}$ indica diferencias significativas entre los grupos expertos e intermedios en la situación estática $[t(18)=3,24, p=0,005]$ y dinámica $[t(18)=2,27, p=0,036]$. Los porteros expertos iniciaron su carrera más cerca del blocaje que los porteros intermedios.

Por último, en lo relacionado con la variable vuelo del balón, la prueba $T$ utilizada como análisis post-hoc muestra diferencias significativas entre los grupos expertos e intermedios en la situación de análisis estática $[t(18)=3,12, p$ $=0,006]$ y entre el grupo intermedios-altos y el grupo intermedios en ambas situaciones: en la situación estática $[t(19)=3,06, p=0,06]$ y en la dinámica $[t(19)$ $=2,46, p=0,024]$. El vuelo del balón fue significativamente mayor cuando los porteros del grupo intermedios trataban de atrapar un balón procedente de un lanzamiento de córner, en comparación con los expertos y los intermedios-altos. 


\section{Datos del comportamiento visual}

Los valores medios (en porcentaje de tiempo mirando) de todos los grupos en el comportamiento visual se presentan en la Figura 2. En la situación estática, el grupo expertos $(n=5)$ obtuvo un porcentaje de tiempo mirando más alto en los dos periodos analizados $(30,72 \%$ en la localización carrera y $34,13 \%$ en la localización balón) en comparación con el grupo intermedios-altos $(n=5)$ $(24,63 \%$ en la localización carrera y $28,22 \%$ en la localización balón) y con el grupo intermedios $(n=5)(23,65 \%$ en la localización carrera y $17,06 \%$ en la localización balón). En la situación dinámica, el grupo intermedios-altos tuvo un porcentaje de tiempo más alto en el porcentaje de tiempo mirando en la localización carrera $(37,4 \%)$ que el grupo de expertos $(29,68 \%)$ y que el grupo intermedio (25,78\%). En la localización balón, los porteros expertos mostraron un porcentaje de tiempo mirando mayor $(34,27 \%)$ que los intermedios-altos $(29,34 \%)$ y que los intermedios $(21,73 \%)$. Las localizaciones relacionadas con los jugadores atacantes obtuvieron valores alrededor de $0 \%$.

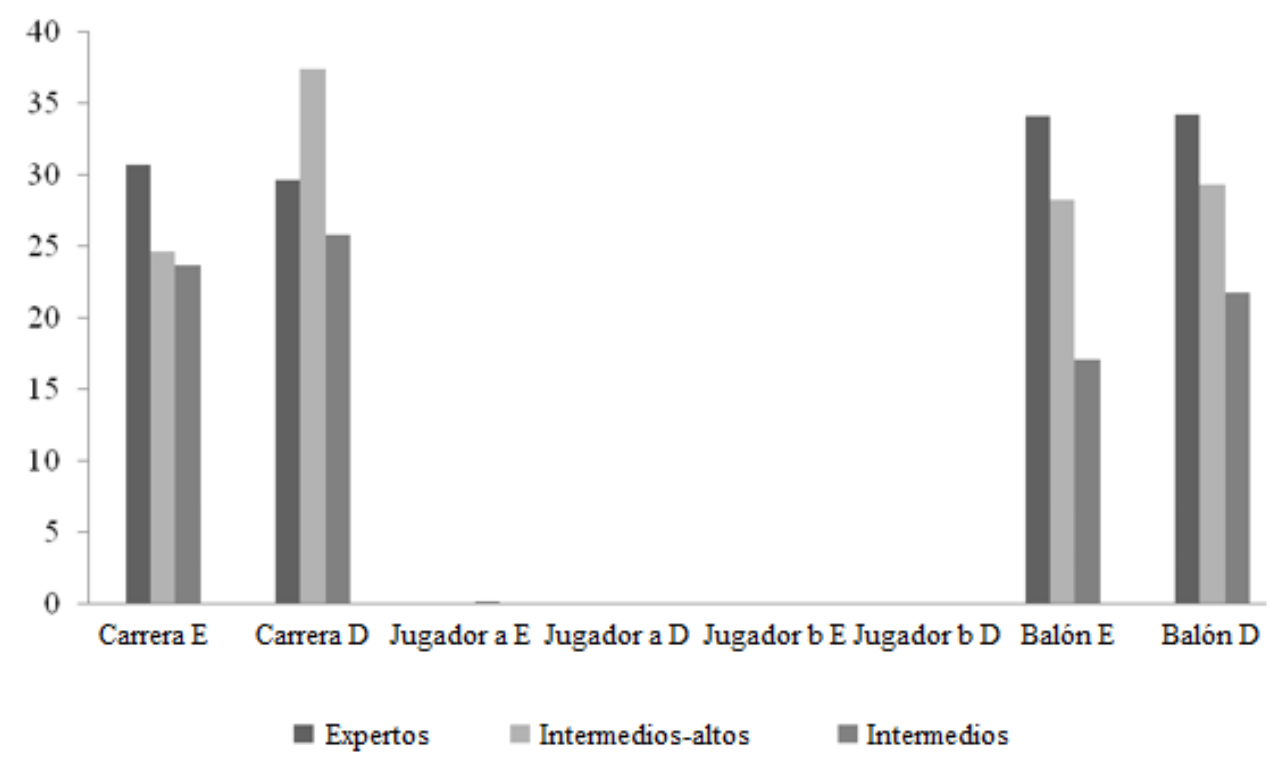

Figura 2. Valores medios del comportamiento visual (en porcentaje de tiempo mirando) para los dos periodos temporales analizados: pre-contacto (porcentaje de tiempo mirando a la localización carrera) y vuelo del balón (porcentaje de tiempo mirando a la localización balón). La letra $E$ después del nombre de la variable indica situación estática y la letra $D$ después del nombre de la variable indica situación dinámica.

El grupo de porteros expertos ha mostrado un porcentaje de tiempo mirando superior al resto de grupos en todas las localizaciones analizadas (excepto en la localización carrera durante la situación dinámica). Sin embargo, el ANOVA de medidas repetidas no ha revelado diferencias significativas entre los grupos en el comportamiento visual.

\section{DISCUSIÓN}

El objetivo fundamental del presente estudio era analizar las diferencias en el comportamiento motor, en el rendimiento y en el comportamiento visual de jóvenes porteros de fútbol cuando tratan de atrapar un balón procedente de un 
lanzamiento de córner. Se ha utilizado el marco de trabajo desarrollado por Newell (1986), manipulando la continua interacción entre los tres limitadores que influyen en el rendimiento (tarea, organismo y entorno).

El grupo de porteros expertos fue más exitoso atrapando balones procedentes de lanzamientos de córner que los otros dos grupos, en las dos situaciones de análisis creadas, confirmándose así la primera de las hipótesis del trabajo. Este rendimiento superior aparece utilizando un patrón de movimiento más preciso y eficiente, esperando durante más tiempo para comenzar la carrera previa, cerca del atrape del balón, y desarrollando toda la acción lo más rápido posible. Los porteros de los grupos intermedios-altos e intermedios iniciaban su carrera previa antes, seguidamente mostraban un patrón coordinativo más lento y, consecuentemente, también realizaban el blocaje más tarde que los expertos. Con este patrón coordinativo, los porteros de los grupos intermedios-altos e intermedios presentaron un porcentaje de fallos significativamente más alto en las dos situaciones analizadas.

El análisis desde el momento del golpeo reveló el comportamiento anticipatorio mostrado por los porteros. Los resultados muestran la tendencia de los porteros expertos de esperar durante más tiempo para comenzar su carrera previa al blocaje. Una estrategia similar fue mostrada por Oudejans, Michaels y Bakker (1997), cuando obtuvieron que los jugadores de béisbol expertos esperaban durante más tiempo a la hora de atrapar bolas de béisbol aéreas, consiguiendo un porcentaje de acierto superior. También en el trabajo previo de Abellán et al. (2016) se mostró que los fallos en el blocaje estaban definidos, entre otros factores, por una excesiva anticipación y un comienzo demasiado temprano de la carrera previa, quizá sin tener suficiente información sobre el destino del balón.

Los porteros de nivel intermedio (intermedios e intermedios-altos) están más afectados por los limitadores del entorno, como se extrae del mejor rendimiento en la situación estática que en la dinámica. El grupo de expertos tiene significativamente menos fallos que los porteros intermedios en las dos situaciones analizadas. Sin embargo, cuando los comparamos con el grupo intermedios-altos, los expertos sólo tienen menos fallos en el blocaje en la situación dinámica. A partir de estos resultados podríamos concluir que los porteros de menor nivel están más afectados (rinden menos) a medida que la tarea se va haciendo más compleja. Estos resultados están de acuerdo con los encontrados en estudios anteriores. Vaeyens, Lenoir, Williams, Mazyn et al. (2007) mostraron que los participantes que no eran jugadores de fútbol fueron menos precisos, en un test de toma de decisiones, que los jugadores de fútbol en todas las situaciones de análisis creadas en ese estudio (2 vs. 1, 3 vs.1, 3 vs. 2,4 vs. 3 and 5 vs. 3). Además, los jugadores de élite y sub-élite fueron mejores que los jugadores regionales en todas las condiciones de análisis, excepto en la situación 2 vs. 1. En un estudio posterior, Vaeyens, Lenoir, Williams y Philippaerts (2007) se clasificó a los participantes en función de su éxito en la toma de decisiones y se obtuvieron resultados similares. Los jugadores más exitosos fueron mejores que los menos exitosos en todas las situaciones analizadas, excepto de nuevo en la situación 2 vs. 1 . El rendimiento y la toma de decisiones de los deportistas varía en función de los limitadores de la tarea 
estudiados (Vaeyens, Lenoir, Williams \& Philippaerts, 2007). El efecto de los limitadores del entorno también ha sido estudiado en el trabajo previo de Abellán et al. (2015). Los porteros analizados presentaban un rendimiento similar al analizar su comportamiento motor durante la tarea de atrapar un balón procedente de un lanzamiento de córner, medido con el porcentaje de aciertos en el blocaje, en dos situaciones de análisis distintas (con y sin atacantes en la zona de acción del portero). Sin embargo, los porteros sí modificaron su patrón coordinativo, adaptándose a las diferentes situaciones de análisis y, consecuentemente, a los diferentes requerimientos de la tarea analizada.

Los hallazgos encontrados en las variables de tiempo de movimiento de las manos, tiempo del salto y tiempo del blocaje son especialmente importantes para el diseño de futuros programas de entrenamiento. Los porteros expertos comenzaron a mover sus manos y a saltar más cerca del contacto con el balón (el blocaje) que los otros grupos (intermedios-altos e intermedios) y, consecuentemente, ellos atraparon el balón antes. Estos resultados son opuestos a los obtenidos en los trabajos previos (Abellán et al., 2016; 2017). En Abellán et al. (2017) el grupo de porteros exitosos obtuvo valores más elevados en la variable vuelo del balón y, por tanto, atraparon el balón más tarde que el grupo de menos exitosos. En Abellán et al. (2016), los fallos se atribuyen a un comienzo del movimiento de las manos demasiado cerca del blocaje. Las diferencias observadas se basan en la presencia o ausencia de jugadores en la zona de acción del portero. Mientras que en los anteriores trabajos de Abellán et al. (2016; 2017) no había jugadores en la zona de acción del portero, en el presente trabajo y en una de las situaciones creadas en Abellán et al. (2015) aparecen dos jugadores en la zona de acción del portero, lo que probablemente tiene un efecto en el comportamiento de los porteros. En un partido real, el portero que es capaz de atrapar el balón antes presenta la estrategia más efectiva, ya que antes termina la posibilidad de anotar un gol por parte del equipo atacante.

Por otra parte, los tres grupos de porteros, durante las dos condiciones analizadas, muestran un comportamiento visual muy similar, caracterizado por un insignificante (muy próximo al $0 \%$ ) porcentaje de tiempo mirando en las localizaciones relacionadas con los jugadores atacantes, lo que indica que esta área no contiene información relevante para los porteros a la hora de realizar el blocaje. El análisis estadístico no muestra diferencias significativas entre los grupos en las situaciones de análisis creadas, lo que no permite confirmar la segunda hipótesis del trabajo. Sin embargo, el grupo de expertos muestra estrategias de búsqueda visual más exhaustivas, con mayor porcentaje de tiempo mirando a las áreas informativas más importantes (la carrera del lanzador en el periodo de pre-contacto y el vuelo del balón en el periodo de contacto con el balón). Los estudios previos han sugerido que el comportamiento visual está determinado por las características específicas de cada situación (Roca, Ford, McRoberts \& Williams, 2013). La idea de que los expertos muestran un comportamiento visual superior, basado en las áreas informativas más importantes, está apoyado por estudios como los desarrollados por Savelsbergh et al. (2002; 2005). Los resultados de las localizaciones relacionadas con los jugadores atacantes (que en ningún caso superan el $0,5 \%$ del porcentaje de tiempo mirando) podrían explicarse con el siguiente argumento: puesto que los 
porteros conocían antes de comenzar el experimento que los jugadores participantes sólo se iban a mover y que no podían tocar el balón, probablemente no los consideraron áreas de relevancia informativa. Por ello, se hacen necesarios más experimentos sobre el comportamiento visual con tareas cercanas a la realidad de competición, con un contacto real entre el portero y los jugadores atacantes durante los lanzamientos de córner, para tratar de obtener una imagen más completa y fiable del comportamiento visual de los porteros durante los lanzamientos de córner.

Teniendo en cuenta los objetivos previstos en la investigación podemos extraer las siguientes conclusiones: Una estrategia exitosa para atrapar un balón aéreo procedente de un lanzamiento de córner consiste en esperar durante más tiempo para obtener más información sobre el vuelo del balón y después comenzar la carrera para tratar de atrapar el balón lo antes posible. Esta ha sido la estrategia mostrada por los porteros expertos, que además han mostrado un rendimiento superior atrapando balones procedentes de lanzamientos de córner.

La sistemática reducción de los datos del comportamiento visual, debido a la realización de test de campo y al aire libre, no permite obtener la suficiente información como para implementar, basándose en estos resultados, un programa de entrenamiento perceptivo para mejorar el rendimiento del blocaje de balones procedentes de lanzamientos de córner. Esta reducción de datos se considera una limitación en el presente trabajo.

Además, aunque la continua interacción de los tres limitadores analizada en esta investigación es la misma que en una acción de juego real, no podemos considerar que realmente la tarea analizada lo sea, ya que no tiene exactamente los mismos requerimientos que un partido real. Debido a la fragilidad del sistema de registro de la mirada, los jugadores situados en la zona de acción del portero no podían intentar rematar el balón, lo que sí que harían en una situación real de juego. Como propuesta de investigación futura, se deberían incorporar jugadores atacantes que intenten conseguir gol, igual que sucedería en un partido real, de manera que así se obtendría una visión más completa de la tarea analizada.

Estos hallazgos deben ser tenidos en cuenta por los profesionales del fútbol, entrenadores y entrenadores de porteros, que tengan como objetivo mejorar el rendimiento de sus porteros. Para ello deben transmitir a los porteros la idea de que no comiencen su carrera antes de obtener suficiente información sobre el destino del balón, así como evitar comenzar la carrera previa en el momento en el que el lanzador del córner golpea el balón.

\section{REFERENCIAS BIBLIOGRÁFICAS}

Abellán, J., Sáez-Gallego, N.M., \& Contreras, O. (2015). Intercepción de un lanzamiento de córner: influencia de los limitadores del entorno. Apunts. Educación Física y Deportes, 122 (4), 61-67. https://doi.org.10.5672/apunts.2014-0983.cat.(2015/4).122.07

Abellán, J., Sáez-Gallego, N.M., Vila-Maldonado, S., \& Contreras, O. (2017). Differences in movement behaviour between successful and less 
successful goalkeepers in the interception of corner kicks. Journal of Human Sport and Exercise, 12(2), 307-315. https://doi.org.10.14198/ihse.2017.122.07

Abellán, J., Savelsbergh, G.P.J., Contreras, O.R., \& Vila-Maldonado, S. (2016). Intercepción de un lanzamiento de córner en fútbol: análisis de la tarea. Revista Internacional de Medicina y Ciencias de la Actividad Física y el Deporte, 16 (61), 111-126. https://doi.org.10.15366/rimcafd2016.61.009

Bernstein, N. A. (1967). The Control and Regulation of Movements. London: Pergamon Press.

Correia V., Araújo D., Duarte R., Travassos B., Passos, P., \& Davids, K. (2012). Changes in practice task constraints shape decision-making behaviours of team games players. Journal of Science and Medicine in Sport, 15, 244249. https://doi.org.10.1016/j.jsams.2011.10.004

Correia V., Araújo D., Vilar L., \& Davids K. (2013). From recording discrete actions to studying continuous goal-directed behaviours in team sports. Journal of Sports Sciences, 31, 546-553. https://doi.org.10.1080/02640414.2012.738926

Davids, K., Williams, A.M., Button, C. \& Court, M. (2001). An integrative modeling approach to the study of intentional movement behaviour. En R.N. Singer, H.A. Hausenblas y C.M. Janelle (Eds.). Handbook of Sport Psychology (pp. 144-173). USA: Wiley.

Handford, C., Davids, K., Bennett, S. \& Button, C. (1997). Skill acquisition in sport: Some applications of an evolving practice ecology. Journal of Sport Sciences, 15, $621-640$.

Headrick, J., Davids, K., Renshaw, I., Araújo, D., Passos, P., \& Fernandes, O. (2012). Proximity-to-goal as a constraint on patterns of behaviour in attacker-defender dyads in team games. Journal of Sport Sciences, 30(3), 247-253. https://doi.org.10.1080/02640414.2011.640706

Newell, K. M., \& Ranganathan, R. (2009). Some contemporary issues in motor learning. En D. Sternad (Ed.). Progress in motor control (pp. 395-404). Springer US. https://doi.org.10.1007/978-0-387-77064-2 20

Newell, K.M. (1986). Constraints on the development of coordination. En M. Wade \& H.T.A. Whiting (Eds.). Motor development in children: Aspects of coordination and control (pp. 341-360). Dordrecht, The Netherlands: Martinus Nijhoff. https://doi.org. 10.1007/978-94-009-4460-2 19

Oudejans, R.R.D., Michaels, C.F., \& Bakker, F.C. (1997). The effect of skill on movement initiation in catching fly balls. Journal of Sport Sciences, 15, 587-595. https://doi.org.1080/026404197367029

Roca, A., Ford, P.R., McRoberts, A.P., \& Williams, A.M. (2013). Perceptualcognitive skills and their interaction as a function of task constraints in soccer. Journal of Sport and Exercise Psychology, 35, 144-155. https://doi.org.10.1123/jsep.35.2.144

Sáez-Gallego, N.M., Vila-Maldonado, S., Abellán, J., \& Contreras, O. (2013). Análisis del comportamiento visual y la toma de decisiones en el bloqueo en voleibol. Cuadernos de Psicología del Deporte, 13(2), 31-44. https://doi.org.10.4321/S1578-84232013000200004

Savelsbergh, G.J.P., Verheul, M., Van der Kamp, J., \& Marple-Horvat, D.E. (2007). Visiomotor control of movement acquisition. En J. Liukkonen (Ed.), 
Psychology for Physical Educators: Student in Focus (pp. 239-260). Leeds: Human Kinetics.

Savelsbergh, G.J.P., Williams, A.M., Van der Kamp, J., \& Ward, P. (2002). Visual search, anticipation and expertise in soccer goalkeepers. Journal of sports sciences, 20(3), 279-287.

Vaeyens, R., Lenoir, M., Williams, A.M., \& Philippaerts, R.M. (2007a). Mechanisms Underpinning Successful Decision Making in Skilled Youth Soccer Players: An Analysis of Visual Search Behaviors. Journal of Motor Behaviour, 39(5), 395-408.

Vaeyens, R., Lenoir, M., Williams, A.M., Mazyn, L., \& Philippaerts, R.M. (2007b). The effects of task constraints on visual search behaviour and decisionmaking skill in youth soccer players. Journal of Sport \& Exercise Psychology, 29, 147-169. https://doi.org.10.1123/jsep.29.2.147

Vila-Maldonado, S., Sáez-Gallego, N.M., Abellán, J., \& Contreras, O. (2012). Efecto del tipo de colocación en el comportamiento visual y la toma de decisiones en bloqueadores de voleibol. Cultura, Ciencia y Deporte, 20(7), 103-114. https://doi.org. $10.12800 /$ ccd.v7i20.56

Vilar, L., Araújo, D., Davids, K., \& Travassos, B. (2012). Constraints on competitive performance of attacker-defender dyads in team sports. Journal of Sports Sciences, 30(5), 459-469. https://doi.org. 10.1080/02640414.2011.627942

Vilar, L., Araújo, D., Davids, K., Correia, V., \& Esteves, P.T. (2013): Spatialtemporal constraints on decision-making during shooting performance in the team sport of futsal. Journal of Sports Sciences, 31(8), 840-846. https://doi.org.10.1080/02640414.2012.753155

Número de citas totales / Total references: 21 (100\%)

Número de citas propias de la revista / Journal's own references: 1 (4,76\%)

Rev.int.med.cienc.act.fís.deporte - vol. 19 - número 74 - ISSN: 1577-0354 